\title{
Computerized maintenance management system agility through receiving PLC feedbacks and examining its effect on maintenance system efficiency
}

\author{
${ }^{1}$ Abass Karami (Corresponding author), ${ }^{2}$ Mohsen Reza Zadeh, \\ IBS degree, Industrial Engineering and Mechanical Engineering, regional CMMS project manager, \\ maintenance expert of Iranian Bothnia steel \\ 2M.A degree, electrical engineering, maintenance expert of Iranian Bothnia steel
}

\begin{abstract}
It is tried in this study to solve the major failures of island-acting based CMMS systems and high dependency to receiving maintenance operative information in equipment functional calendars through creating a one-way link from PLC monitoring computer with server software of CMMSwith the lowest cost and finally, its effects on defects caused by the mentioned faults would be analyzed.The sample context is Kerman's oil warehouse project. Desk study has been used and the information recorded in CMMS software has been utilized as resources for collecting statistical information to analyze efficiency of the practical activities performed. After examining normalization of the tests distribution using Kolmogorov-Smirnov test, analyzing the data using two dependent samples average comparison test (paired t-test) was conducted through SPSS software for each of the surveying components in situations of before and after the executive process. The findings indicate that creating a link between PLC and CMMS in addition to continuous update of the equipment function calendar concomitant with receiving constants feedbacks from PLC system reduce the available equipping time by decreasing the failures and considerably increase access to the repair personnel and also heighten the capability ofbetter managing periodic services consumables.
\end{abstract}

Keywords: CMMS, computerized maintenance management system, industrial automation, PLC

\section{Introduction}

It has been a long time that advanced industrial countries have passed traditional repairing periods to modern servicing and maintenance. Furthermore, developing countries tend to better utilization of physical properties (Bagadia, 2006). Maintenance is a complicated process beginning with equipment fault or doing a planned repair. This process needs planning, timing, controlling, and using repairing tools for performing important maintenance activities(Sohal \&Schoeman,2010). In an applied language, maintenance system is a set of instructions, methods, processes, software and hardware facilities used for repairing and maintaining the organization machines and equipment in an acceptable and standard situation (Ajorloo, 1997).

Maintenance and repairing activities are divided into two categories generally:

Unplanned activities: There processes include emergency maintenance and random technical inspections. Naturally in this method, there is no prior planning for fault recognition and the maintenances are merely done after a failure occurs. (Samandarizadeh, 2010).

Planned activities: This type of activities encompasses a key and major portion of maintenance strategies. They are done previous to failure and for preventing or planned for forecasting accidental errors (Such as maintenance activities and turn technical inspections or PM, calibration, and activities relating to predictive maintenance (PDM) and condition-based maintenance (CBM) methods including oil sampling for the device status analysis) or they are performed after a fault for modifying the relating subsystems which these activities are called corrective maintenance (CM) (Hekmat, 2003).

\section{Periodic frequency in planned activities}

In the field of inspection processes preventive maintenance, adjusting, replacing, and repairing of the parts and machines are periodically performed and they are scheduled (Alborz Tadbir, 2014).

\section{CMMS}

The information volume increase in maintenance units and necessity to analyze data for appropriate decision making have revealed the demand for maintenance automation. 


\section{A CMMS must have the following components at least:}

Equipment management (Physical properties)

Emergency maintenance requests management

Corrective maintenance requests management

System definitions bank

Managing the preventive maintenance activities, planning and scheduling, and the functional calendar.

Report system

The general method for planning the periodic activities in CMMSs is creating a specific function calendar for each piece of the equipment so as the equipment days and working hours are defined and periodic service requests are proposed based on calendar counting algorithm. Thus, particular calendars should be designed for the pieces of equipment which do not have 24-hour working time which considerably increases the software definition and deployment period and heightens management problems in the utilization period, subsequently.

This study is aimed at presenting acheap and reliable method based on industrial automation in order to reduce CMMSs periodic and functional calendars faults and finally, it would be executed in a sample space and its implementation findings would be analyzed using statistical methods. Regional web-based CMMS written by PHP language and MySQL data engine was used in this survey.

\section{PLC}

The origin of industrial automation with machines and its control processes gets back to 1920s by emergence of initial pneumatic controllers. It has been 3 decades from the first years of PLC birth. Throughout this period, we have seen numerous changes in PLC structure including performance speed increase, the capability of working with analog and digital signals, and possessing fast communication facilities.

Specific programmers were required for programming old PLCs which would lead to high price of a logic control system with PLC.Currently, it is feasible to program PLCs using personal computers which causes significant simplicity and savings.

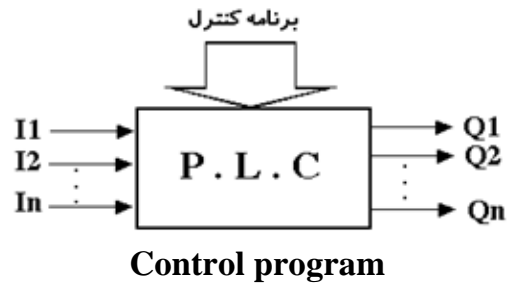

Each PLC has its own specific programming language which is the interface between user and PLC software. Using control software, the PLC can manage the desired process. The most important methods for PLC programming are ladder, flowchart, and statement listing. In recent years, toward improving and facilitating the working with PLC systems, some situations have developed that provided programming and the use of graphical environment for easier utilization of industrial automation systems. Human-machine interfaces (HMIs) are a prominent example of graphical interface for PLC industrial automation systems. PLC system used in this article is PLC Step 7 and the software used for planning and programming the mentioned PLC is WinCC. Recently, with the advent of OPCs (OLE for process control) as the providers of PLC utilization platforms for organizations software systems, computerized monitoring situations for software systems such as ERPs have reached high levels of capability.

\subsection{Establishing a link between CMMS and PLC}

The use of OPS servers mostly means the necessity of designated CMMS programming and specific ERPS in higher levels for the user organizations. The information available in industrial automation systems can be the feed of the organization software systems including CMMSs. All the data sent to electro motors, fans, blowers, and other industrial equipment in addition to all the information received from sensors, detectors, and local feedback systems in the industrial automation system can be received and used.

\subsection{Research method}

This practical research is conducted through samples in Kerman oil warehouse and since all confounding factors such as personnel quality errors in performing services and so forth have not changed in computerized and manual situations, the results are examined using paired t-test for the equipment fault components, consumables required for services, manpower needed for registering information and servicing the 
equipment quantitatively in a 4-month interval after performing practical activities on 40 pieces of equipment. The findings will be survey in the followings.

Equipment breakdowns: Computer reliability for recording the equipment working data in seconds and removing the working data registering errors and so forth in addition to subsequent CMMS performance according to this information lead to expecting that services would be given exactly in the equipment real moment with high possibility which should result in fewer equipment faults.

Consumables required for periodic services: It is expected that services consumables utilization would be reduced in line with manufacturers recommendations written in equipment service and maintenance instructions.

Manpower demanded for information registration:It is supposed after performing the project that the amount of person-hour of this set of personnel gets close to zero.

Manpower needed for equipment service: It is expected after performing the project that the person-hour amountof servicers working would be very near to what estimated by maintenance planning experts.

Table 1: Check Normality of amount of equipments failures

\begin{tabular}{|c|c|c|c|c|}
\hline $\mathrm{p}$ & Z Kolmogorov-Smirnov & $\mathrm{SD}^{1}$ & mean & variable \\
\hline 0.227 & 1.043 & 1.8 & 3.3 & Equipment failures \\
\hline
\end{tabular}

Kolmogorov-Smirnov test is not significant for the number of equipment faults $(p=0 / 555)$, thus the number of equipment errors has a normal distribution and parametric analyses can be used for that.

Table 2: Check the normality of amount of material for periodic services

\begin{tabular}{|c|c|c|c|c|}
\hline $\mathrm{p}$ & Z Kolmogorov-Smirnov & SD & mean & variable \\
\hline 0.118 & 1.188 & 0.964 & 2.472 & Material required for periodic services \\
\hline
\end{tabular}

Kolmogorov-Smirnov test is not significant for the amount of periodic services consumables $(\mathrm{p}=0.118)$. Therefore, the amount of materials used for periodic services has a normal distribution and parametric analysis can be utilized for that.

Table 3: Check the normality of the man-hours for recording information.

\begin{tabular}{|c|c|c|c|c|}
\hline $\mathrm{p}$ & Z Kolmogorov-Smirnov & SD & Mean & Variable \\
\hline 0.468 & 0.848 & 2.649 & 7.44 & Manpower required for recording information \\
\hline
\end{tabular}

Kolmogorov-Smirnov test is not significant for the amount of person-hour of manpower required for data registration $(p=0 / 468)$. As a result, the person-hour amount of human resources needed for data recording has a normal distribution and parametric analyses can be used for that.

Table 4: Effect of a one-way link between PLC and CMMS on equipment failures

\begin{tabular}{|r|r|r|r|r|r|}
\hline p-value & $\mathbf{t}$ & $\mathbf{S D}$ & Mean & Phase & Variable \\
\hline 0.000 & \multirow{2}{*}{6.553} & 1.800 & 3.38 & Before the run & equipment failures \\
\cline { 3 - 5 } & & 1.159 & 1.88 & after the run & \\
\hline
\end{tabular}

Breakdowns average of 3.38 previous to performing the project has been decreased to 1.88 after the project execution and this reduction is statistically significant $(\mathrm{p}=0 / 000)$.

Table 5: Effect of PLC data link with CMMS on material required for services

\begin{tabular}{|c|c|c|c|c|c|}
\hline p-value & $\mathbf{t}$ & SD & Mean & Phase & Variable \\
\hline 0.000 & 8.10 & 0.964 & 2.472 & Before the run & Amount of material \\
& & 0.902 & 1.45 & After running & for periodic services \\
\cline { 3 - 5 } & &
\end{tabular}

Likewise, consumables average for periodic services has been decreased until 1 unit which this reduction is statistically significant $(\mathrm{p}=0 / 000)$.

\footnotetext{
${ }^{1}$ The standard deviation
} 
Computerized maintenance management system agility through receiving PLC feedbacks ...

Table 6: Effect of PLC data link with CMMS on manpower required

\begin{tabular}{|c|c|c|c|c|c|}
\hline p-value & $\mathbf{t}$ & SD & Mean & Phase & Variable \\
\hline 0.000 & 15.545 & 2.648 & 7.43 & Before the run & $\begin{array}{c}\text { Human resources required for } \\
\text { servicing }\end{array}$ \\
\cline { 3 - 6 } & & 0.563 & 1.7 & After running \\
\hline
\end{tabular}

The average of manpower needed for registering equipment functional information has been decreased until 4.5 times after executing the project which this reduction is statistically significant.Regarding the analysis of the PLC and CMMS information link effect on the amount of human resources required for giving periodic services, the estimated time average for giving every service is 5.384 person-hour which is considerably close to the maintenance planning unit estimated amount while it was 6.784 before performing the project. This issue indicates a more realistic terms of service for use of human resources.

\section{Findings and conclusion}

The results of the scientific and practical activity done in this study represents that by low cost, the method of relative integration of CMMS systems is an efficient strategy for organizations with industrial automation infrastructures based on PLC which will lead to costs reduction, equipment reliability increase, and increasing the employees' reliability to the maintenance system performance as an independent tool. Furthermore, as it can be seen, from the situation before performing the project to after executing it, the average of faults, the average of materials used for periodic services such as oil, grease and so on, and the amount of manpower needed for registering the equipment working information in CMMS software have been changed from 3.38 to $1.88,2.475$ to 1.457 , and 7.43 to 1.7 , respectively. All of these data are significant at $\mathrm{p}<0.005$ level. Thus, trying to implement such processes in large industrial systems would be considerably efficient in improving the performance of maintenance organization particularly in optimizing the resources used in doing periodic services without the need for fundamental changes in structure of the software used in the organization like CMMSs or PLC industrial automation systems.

\section{References}

[1]. Kris Bagadia, Computerized Maintenance Management Systems Made Easy, 2006

[2]. Simon, A. \& Schoeman, P. \& Sohal, A. (2010). Prioritised best practices in a ratified consulting services maturity model for ERP consulting. Journal of Enterprise Information Management, 23(1), 100 - 124

[3]. Bhatt, G. \& Gupta, N. \& Kitchens, F.(2010). An exploratory study of groupware use in the knowledge management process. Journal of Enterprise Information Management, 18(1), 28 - 46. 